\title{
COMMENT
}

ENERGY Heed those who witnessed the Chernobyl nuclear disaster $\mathbf{p . 4 5 0}$
BONES The story of our eerily fascinating skeleton is one of endurance $\mathbf{p . 4 5 2}$
SEXISM Hunt for brain differences is a lesson in bad science $\mathbf{p . 4 5 3}$
GENE EDITING An Islamic perspective on the CRISPR twins born in China $\mathbf{p . 4 5 5}$

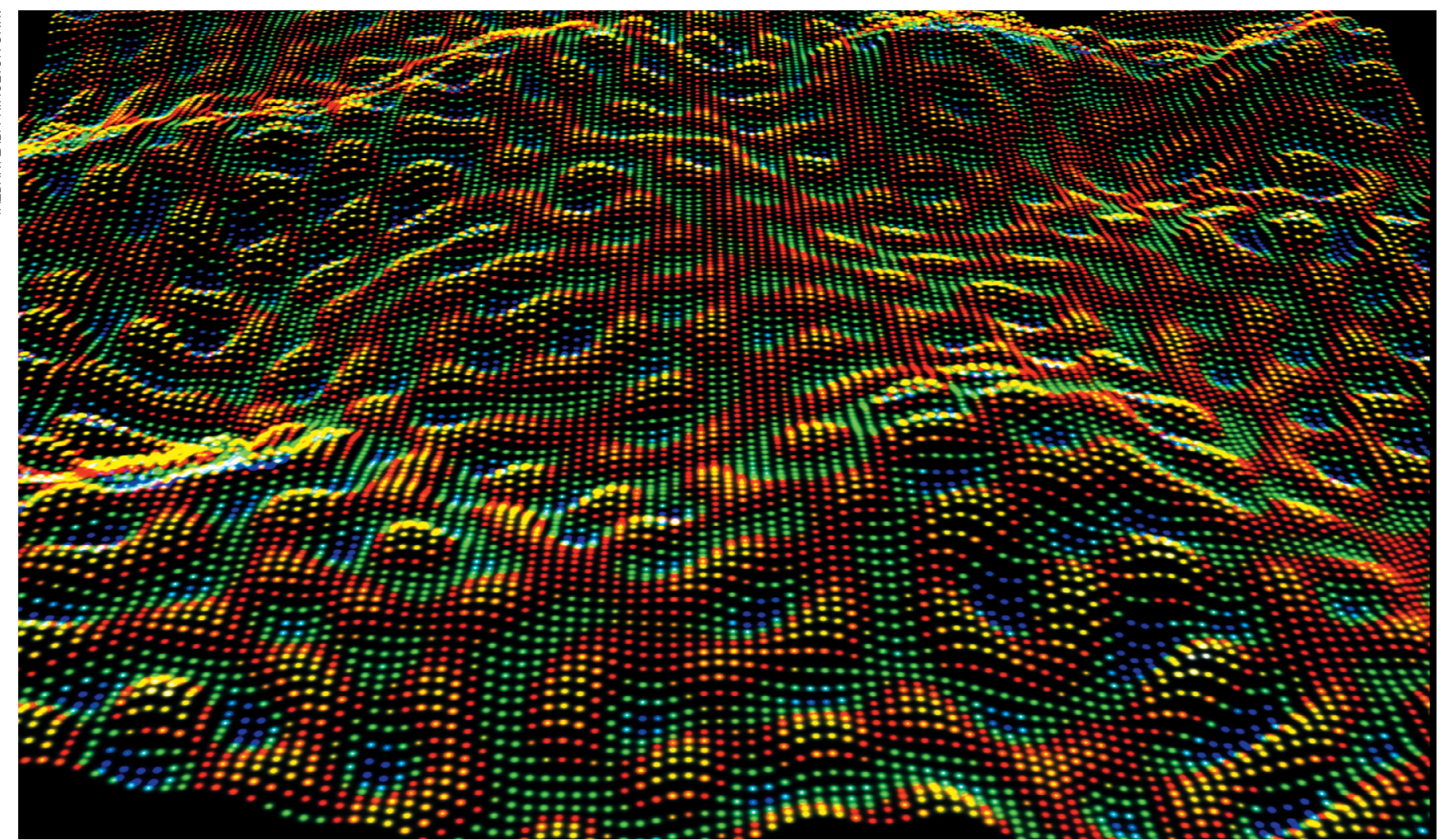

The surface of a topological insulator, as seen under a scanning tunnelling microscope.

\section{Beware plausible predictions of fantasy materials}

Real materials rarely behave as models suggest. Stability must be part of the burden of proof for predicting the complex properties of matter, urges Alex Zunger.

$\mathrm{T}$ The hunt is on for materials that have exotic properties, to enhance quantum computers, touch screens and electronic displays, and to double the efficiency of solar cells, for example. Historically, materials that revolutionized technology, including tungsten light-bulb filaments, penicillin, Teflon and $\mathrm{C}_{60}$ buckyballs, were found through a combination of intuition, trial and error and lucky mishaps. But the complex characteristics needed today cannot be found by accident: they must be designed in at the start.
Materials are generally defined by the atoms that make them, and the structures and compositions that these atoms take up. Properties of materials - such as colour, hardness, conductivity, stability, topology or magnetism - exist in certain substances and not others. The question is, how can we find materials with just the right properties we dream of?

The emergence of radically new forms of matter will transform our understanding of basic physics. Such forms include materials with topological properties that give rise to unusual electronic features - these stem from the underlying symmetries of the positions of atoms. Other forms, such as quantum spin liquids and new types of superconductor, could also transform technology. The most obvious materials with simple properties were found decades ago. Today, we must dig much deeper into the space of possible materials to find new gold.

Theoretical physicists are now using computers to model and screen thousands of candidate materials that might have exotic 
properties. But the latest wave of predicting topological compounds considers only combinations of atoms that have a certain property (see 'Tougher screening'), which don't necessarily form stable compounds. Many hypothetical materials turn out to be unstable and impossible to synthesize.

Theorists who design materials must add extra filters to avoid sending their chemistry colleagues off to the lab on a pointless quest. Focusing on current strategies for predicting topological materials ${ }^{1-5}$, here I illustrate the problems by describing four common ways in which predictions might fail, offering exciting properties in unstable materials. Extra calculations can narrow down lists of intriguing materials to those that are most likely to be stable and realizable.

\section{MATTER BY DESIGN}

Finding materials that host specific properties ${ }^{6}$ follows a process known as 'inverse design'. First, pick a target property (say, thermoelectricity, topology or superconductivity) and a theoretical framework in which to calculate it for a given arrangement of atoms in a crystal. Second, search for stable crystal structures that host such properties. Quantum mechanics, in the form of the Schrödinger equation, provides the bridge between these steps.

Examples of new materials that have been predicted in this way, and whose properties have been confirmed in the lab, include thermoelectric compounds (that combine normally contradictory properties of thermal insulation and electrical conductivity) and transparent conductors for use in displays and solar cells - an unlikely combination of properties, given that conductors are generally opaque and insulators normally transparent. Close collaboration was crucial between the theorists who predicted these materials and the experimentalists who made and validated them.

The discovery of the topological behaviour of compounds is one of the most exciting recent developments in condensedmatter physics and solid-state chemistry ${ }^{7}$. Symmetries in the positions of atoms and properties of the electrons in the crystal - such as time-reversal symmetry (for topological insulators), mirror symmetry (for topological crystal insulators) or rotational symmetry (for topological Dirac semimetals) - give these materials unusual electronic properties. Topological insulators, for example, do not conduct electricity in their bulk. Yet, on their surfaces, they can host special 2D conductive metallic states, through which electrons can flow without scattering. These raise hopes for new electronics that draw current without dissipation and, in combination with superconductors, for building a practical quantum computer.

Disappointingly, so far only a handful of topological materials have been made and their properties verified. Their structure

and compositions were initially conceived intuitively, and then a range of 'usual suspect' compounds tested. In the past year or so, some researchers ${ }^{1-3}$ have extended such ideas to identify the organization of atoms in crystals that seem likely to deliver topological properties, using a version of matter-by-design. The first step taken ${ }^{1-3}$ was to identify symmetries that classify the topology of the compound (whether it is a topological insulator or a Dirac semimetal). The second step was searching databases of compounds ${ }^{1-3}$ and computed electronic properties ('band structure') looking for materials that satisfy the topological classifications. This process (see 'Tougher screening', part A) has revealed tens of thousands of topoloids. Researchers anticipate a wave of exciting experimental synthesis and characterization of new topoloids ${ }^{8}$.

\section{NOT SO FAST}

But this will not be so easy in practice. The described procedure applies equally to real or unreal compounds; not all will be feasible to make or have their properties forecast. Theoretical predictions are only as good as the ingredients that go into them. And theorists do not always ask all the important questions. For instance, are the presumed crystal structures stable and synthesizable? Are the electronic band structures used correct? Indeed, some topological properties work against stability. For example, designing a topological insulator requires rearranging the order of some

quantum states while moving some electrons from bound ('chemically happy') quantum states to less favourable ('chemically unhappy') ones.

\section{"Extrafilters would protect condensed- matter theory from complaints of frivolous predictions."}

Bonding is weakened, and the lattice destabilized. The crystal might spontaneously alter the positions of its atoms to compensate. And the new atomic positions might no longer have the all-important symmetries.

Many other practical factors are also not considered in theoretical searches. Databases of experimental or calculated chemical properties often do not account for spontaneous crystal defects, magnetism or other chemical elements that must be inserted (doped) into the solid to increase the supply of electrons. Computational models assume that materials have simple atomic structures, with atoms in ideal locations and no magnetism. They often use averaged configurations of atoms, not actual ones.

Fortunately, many of these instabilities can be explored using quantum calculations on real solids (see 'Tougher screening', part B). For example, stability can be examined by calculating the total energy of electrons and ions in the given configuration; the lower the energy, the firmer the chemical bonds. Variants of the modelled structures can be tested by 'nudging' atoms, spins or electrons and evaluating the total energy of each perturbed structure.

Applying extra filters such as these to material selection would help to avoid falsepositive predictions. It would also protect the brand of condensed-matter theory from complaints of frivolous predictions, and strengthen cherished theory-experiment collaborations.

\section{FOUR FILTERS}

The following failure modes leading to false-positive predictions are common.

Thermodynamic instability. These are cases of mistaken identity - the assumed structure is not the lowest-energy arrangement of the atoms. If the hypothetical topological structure is thermodynamically unstable, the symmetry that confers the magic properties might be lost. Physicists love simple structures, such as honeycomb or triangular lattices, even though these are rare in the real world. They can make hypothetical structures easily in the computer for certain atoms, but in the real world, such atoms might not stay put. For example, the first predicted Dirac semimetal in three dimensions, bismuth dioxide, was assumed to have a structure similar to that of silicon dioxide $(\beta \text {-cristobalite })^{9}$, even though that is never seen in bismuth compounds. But energy calculations ${ }^{10}$ reveal this not to be the case - the bismuth and oxygen atoms shift so that the $\mathrm{BiO}_{2}$ lattice has lower energy. The resulting real compound does not behave like a Dirac semimetal. Dozens of other such examples of predicted topological attributes in unstable structures can be found in the topological literature ${ }^{10}$.

Defects that form spontaneously. Solids are often imperfect. Defects and impurities are often introduced inadvertently during manufacturing. But in some materials, atoms are ejected spontaneously from their positions in the lattice, to relieve crowding and lower the crystal's energy. Such materials are 'born' with defects, which can break the symmetry of the lattice, killing topological behaviour. For example, in 2016, the compound tetrabarium tribismuth $\left(\mathrm{Ba}_{4} \mathrm{Bi}_{3}\right)$ was predicted ${ }^{4}$ to have a new type of topological state, with no analogue in high-energy particle physics but with potential uses in quantum computing. However, this prediction assumes an ideal crystal structure. Calculation of the quantum states of this material ${ }^{10}$ shows missing electrons ('holes') in its normally occupied states. But shifting Bi atoms out of their positions is rewarded chemically by lowering the energy of this crystal - it releases electrons that fill the holes. This process is predicted to create organized arrays of $\mathrm{Bi}$-atom vacancies in the lattice, thus altering its underlying symmetry. Experiments are still needed to examine the 


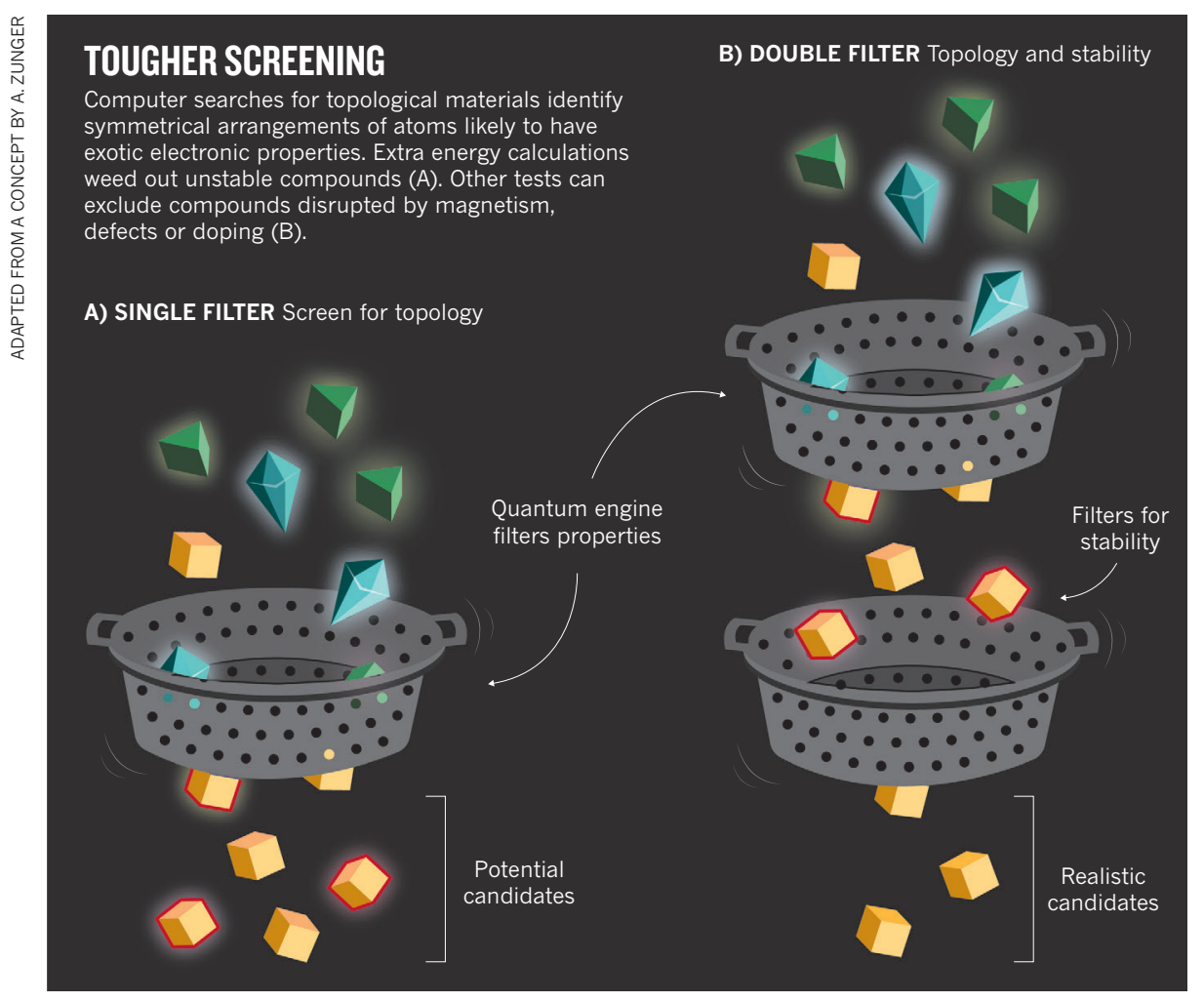

real structure of this compound. But this case exemplifies a broader category of would-be topological materials in which the natural formation of defects can be fatal to topology.

Magnetism that converts a metal to an insulator. Experimental and computational databases often do not say whether a structure is magnetic or not. But magnetism can alter the energy states and symmetries of a material, and thus its topological properties. For example, the assumed non-magnetic structure of copper dibismuth tetraoxide $\left(\mathrm{CuBi}_{2} \mathrm{O}_{4}\right)$ was predicted to have exotic topological properties enabled by its unusual pattern of overlapping metallic energy bands. However, this assumption might not always hold. Its copper ions have an odd number of electrons. These can manifest unpaired electron spins, thus making the material magnetic and insulating, and wiping out the electronic band structure predicted for the ideal form.

This can be foreseen: computing the total energy (in antiferromagnetic as well as paramagnetic configurations) lowers the energy enormously, converting the compound from a metal to an insulator to make it more stable ${ }^{10}$. Experiments confirm that the material is an insulator even at room temperature, at which it is typically used as a photocatalyst (this function builds on it being a non-metal). One would expect that further structures are vulnerable to developing spontaneous magnetism, defeating the high symmetry that yields topology.

When doping is needed but destroys the crystal. Some compounds, such as cubic barium bismuth trioxide $\left(\mathrm{BaBiO}_{3}\right)$, are predicted to be topological - but only in their electronic excited state, not in their normal ground state ${ }^{5}$. For example, replacing one-third of the oxygen atoms in $\mathrm{BaBiO}_{3}$ with fluorine, which is known to release electrons into the material (doping), was thought to populate some high energy levels with enough electrons to achieve topology. But in reality, moving electrons into the higher energy states could actually break up the crystal. To examine such intuitions, my colleagues and I calculated the total energy of the compound and its likely decomposition products, finding that $\mathrm{BaBiO}_{2} \mathrm{~F}$ would not exist, but would break down to $\mathrm{Bi}_{2} \mathrm{O}_{3}, \mathrm{Ba}_{2} \mathrm{Bi}_{2} \mathrm{O}_{5}$ and $\mathrm{BaF}_{2}$ with much lower energy ${ }^{10}$ and no topology. Because extreme doping alters the structures of crystals, some predicted compounds that require doping to make them topoloids might fail to be realized in the lab.

Materials scientists cannot tell yet how many of the predicted thousands of topological properties do not exist in realizable materials. These four failure modes are unlikely to be the only ones. Artificially high symmetries might also be impossible to make in practice. Some materials can break symmetry and take on different local motifs that often have lower symmetry than the average structure. Theorists like to average these motifs out, and so might miss or mistake cases of topology.

\section{NEXT STEPS}

The action needed is straightforward, if computer-intensive: assumed structures can be perturbed by 'nudging' atoms or spins or electrons, then evaluating the total energy of competing configurations, examining whether the low-energy ones still possess the topology-enabling symmetry of the originally assumed structure. Theorists should do more to check that instabilities like these - that can be foreseen at the outset - do not break the precious symmetries that give topological materials their unconventional properties. Such basic checks should be made using the matter-by-design approach before hypothetical compounds are sent for experimental evaluation. The symmetry-breaking effects listed here are not gentle or subtle they change the very nature of the material.

Theorists should even question the accuracy of structures listed in experimental or theoretical databases. Spontaneous defect formation is often missed, and, in many cases, the structure listed corresponds to some idealized, symmetrical average structure. Yet it is usually lower forms of symmetries (such as motifs in polymorphous structures) in specific atomic arrangements (unaveraged) that really decide topology. Examples of polymorphous networks whose symmetries need greater understanding include cubic halide perovskites, paramagnets and paraelectric oxides. Theoretical databases often do not specify magnetism, or the consequences of doping. Contributing scientists should be mindful of these details and fill gaps.

The next step to improve materials models is to develop calculations of perturbed lattice structures and competing phases, in search of stable and synthesizable compounds. This will entail closer interactions between experimentalists and theorists. Databases must be expanded to include the effects of polymorphous structures, defects, magnetism and doping on the basic symmetries of compounds.

Theories must shift, from describing possible properties in hypothetical structures to revealing real structures that can host exotic properties with impunity.

Alex Zunger is a theoretical condensedmatter physicist at the Renewable and Sustainable Energy Institute (RASEI), University of Colorado, Boulder, USA. e-mail:alex.zunger@colorado.edu

1. Zhang, T. et al. Preprint at https://arxiv.org/ abs/1807.08756 (2018).

2. Vergniory, M. G., Elcoro, L., Felser, C., Bernevig, B. A. \& Wang, Z. Preprint at https:// arxiv.org/abs/1807.10271 (2018).

3. Tang, F., Po, H. C., Vishwanath, A. \& Wan, X. Preprint at https://arxiv.org/abs/1807.09744 (2018).

4. Bradlyn, B. et al. Science 353, aaf5037 (2016).

5. Yan, B., Jansen, M. \& Felser, C. Nature Phys. 9 709-711 (2013).

6. Zunger, A. Nature Rev. Chem. 2, 0121 (2018).

7. Qi, X.-L. \& Zhang, S.-C. Phys. Today 63, 33-38 (2010).

8. Nature 560, 151-152 (2018)

9. Young, S. M. et al. Phys. Rev. Lett. 108, 140405 (2012).

10.Zunger, A., Malyi, O., Dalpian, G. M., Zhao, X. \& Wang, Z. Preprint at https://arxiv.org/ abs/1812.10573 (2018). 